\title{
Statistical analysis of the two stage mutation model in von Hippel-Lindau disease, and in sporadic cerebellar haemangioblastoma and renal cell carcinoma
}

\author{
E R Maher, J R W Yates, M A Ferguson-Smith
}

\begin{abstract}
Analysis of the age incidence curves for unilateral and bilateral retinoblastoma led Knudson to propose that hereditary tumours may arise by a single event and sporadic tumours by a two stage mutation process. It has been suggested recently that sporadic renal cell carcinoma may arise from a two stage mutation process. We analysed the age incidence curves for symptomatic renal cell carcinoma $(n=26)$ and cerebellar haemangioblastoma ( $n=68)$ in 109 patients with von HippelLindau (VHL) disease, and compared them to 104 patients with sporadic renal cell carcinoma and 43 patients with sporadic cerebellar haemangioblastoma. The age incidence curves for renal cell carcinoma and cerebellar haemangioblastoma in VHL disease were compatible with a single mutation model, whereas the age incidence curves for sporadic renal cell carcinoma and cerebellar haemangioblastoma suggested a two stage mutation process. These data are compatible with the VHL gene functioning as a recessive tumour suppressor gene. Sporadic cerebellar haemangioblastoma and some renal cell carcinoma may arise from somatic mutations inactivating both alleles at the VHL locus.
\end{abstract}

Knudson ${ }^{1}$ suggested that human cancers could arise by a two stage mutation process. The first mutation could arise in either germline or somatic tissue, but the second event was always in the latter. Statistical analysis of the age incidence of unilateral retinoblastoma tumours (most of which are sporadic) showed a pattern expected if two independent

Cambridge University Department of Pathology, Tennis Court Road, Cambridge.

E R Maher, J R W Yates, M A Ferguson-Smith

Correspondence to Dr Maher, Department of Medical Genetics, Level 1, Addenbrooke's Hospital, Hills Road, Cambridge CB2 2QQ.

Received for publication 21 September 1989.

Revised version accepted for publication 12 December 1989. somatic mutations were necessary for a tumour to develop. $^{1}$ In contrast, bilateral retinoblastoma tumours (all of which are inherited) behaved as if only one somatic mutation was necessary, and it was suggested that the first mutation is an inherited germline event. The second mutation is somatic and may occur by a variety of mechanisms including chromosome loss (with or without reduplication), deletion, mitotic recombination, gene conversion, or point mutation. ${ }^{2}$ The retinoblastoma gene thus functions as a recessive 'tumour suppressor gene' and this model has become a paradigm for the study of inherited cancer syndromes and their sporadic counterparts.

Statistical analysis of age at diagnosis rates for inherited and sporadic cases of Wilms' tumour, ${ }^{3}$ neuroblastoma and phaeochromocytoma, ${ }^{4}$ and medullary cell carcinoma ${ }^{5}$ gave similar results to those in retinoblastoma. ${ }^{1}$ Recently, Erlandssen $e t a t^{6}$ analysed the age incidence of renal cell carcinoma and found evidence that sporadic tumours arise from a two stage mutation process whereas inherited renal cell carcinomas require only a single somatic mutation as in inherited retinoblastoma cases. Von HippelLindau (VHL) disease is an autosomal dominant disorder with a predisposition to develop a variety of tumours, most frequently haemangioblastoma of the retina, cerebellum, and spinal cord, renal cell carcinoma, and phaeochromocytoma. ${ }^{7-9}$ Although renal cell carcinoma has been said to occur in up to $40 \%$ of VHL patients, ${ }^{10}$ it appears that most affected subjects will develop renal cell carcinoma if they live long enough. ${ }^{11}$ Patients with VHL disease were not included in the study by Erlandssen et al. ${ }^{6} \mathrm{We}$ have therefore analysed the age at diagnosis of cerebellar haemangioblastoma and renal cell carcinoma in VHL disease, and compared it to that of sporadic tumours to determine if there is statistical evidence for a two hit mutation process occurring in these VHL disease tumours and their sporadic counterparts.

Patients and methods

Patients with VHL disease were ascertained for the purpose of molecular genetic studies by contacting 
medical specialists throughout Great Britain. Clinical details were obtained on 109 patients (eight sporadic, 101 familial from 37 kindreds), of whom 68 had a cerebellar haemangioblastoma and 34 a renal cell carcinoma. All patients with cerebellar haemangioblastoma were symptomatic at diagnosis. However, eight cases of renal cell carcinoma were diagnosed in asymptomatic subjects and these were excluded from the analysis as they would bias towards an earlier age at diagnosis in VHL disease patients. Control patients with sporadic (non-familial) tumours were ascertained from records of the Department of Histopathology at Addenbrooke's Hospital. Forty-three patients with sporadic cerebellar haemangioblastoma were diagnosed between 1977 and 1989, and 104 patients with renal cell carcinoma (clear cell or adenocarcinoma) were diagnosed from 1980 to 1988.

Statistical analysis of tumour age incidence was performed according to the method of Knudson. ${ }^{1}$ The proportion of patients not yet diagnosed was plotted against age ( $t$ ). For a single stage mutation process, as seen in bilateral retinoblastoma, a first order equation of the form $\log \mathrm{S}=\mathrm{a}-\mathrm{kt}$ is expected. For a two hit mutation process as occurs in unilateral (sporadic) retinoblastomas a second order equation in the form $\log \mathrm{S}=\mathrm{a}-\mathrm{kt}^{2}$ is expected. For each tumour type the data were analysed to determine if a linear relationship (first order) or curvilinear relationship between $\log S$ and $t$ was apparent. Linear regression lines were calculated according to the least squares method. Unpaired $t$ testing and $\chi^{2}$ test (with Yates's correction) were applied as necessary. Statistical significance was taken at the $5 \%$ level.

\section{Results}

\section{CEREBELLAR HAEMANGIOBLASTOMA}

The age distribution of cerebellar haemangioblastomas is shown in fig 1. Mean age at diagnosis in VHL disease was significantly younger than in

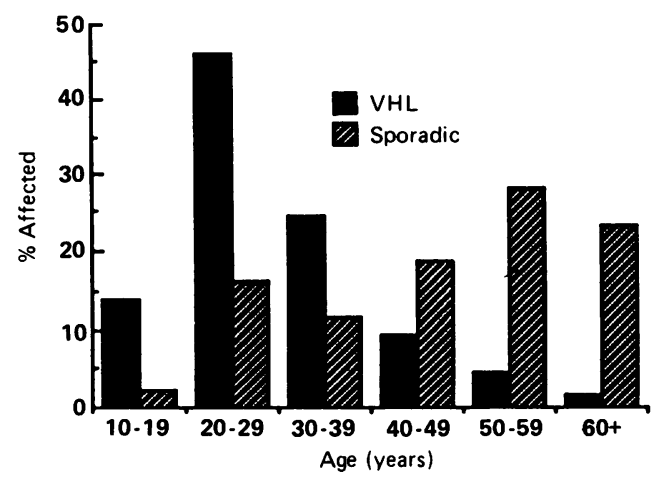

Figure 1 Age distribution of cerebellar haemangioblastoma in sporadic cases and in patients with VHL disease. sporadic cases $(29 \cdot 0$ (SD 10.1) versus $47 \cdot 7$ (SD 14.9) years, $t=7 \cdot 24, \mathrm{p}<0.001)$. The relationship between proportion of cases not yet diagnosed (S) and age for VHL disease tumours followed a single order equation (fig 2). In contrast, for sporadic cases the data fitted a second order equation.

\section{RENAL CELL CARCINOMA}

The age distributions of renal cell carcinoma are shown in fig 3. The mean age at diagnosis of renal cell carcinoma in VHL patients with symptomatic renal cell carcinoma was significantly younger than those with sporadic tumours $(44 \cdot 8$ (SD 9.6) versus $61 \cdot 8$ (SD $10 \cdot 8$ ) years, $t=7 \cdot 3, \mathrm{p}<0.001)$. An excess of males was apparent in sporadic cases ( 72 males, 32 females), but not in VHL disease patients (13 males, 13 females).

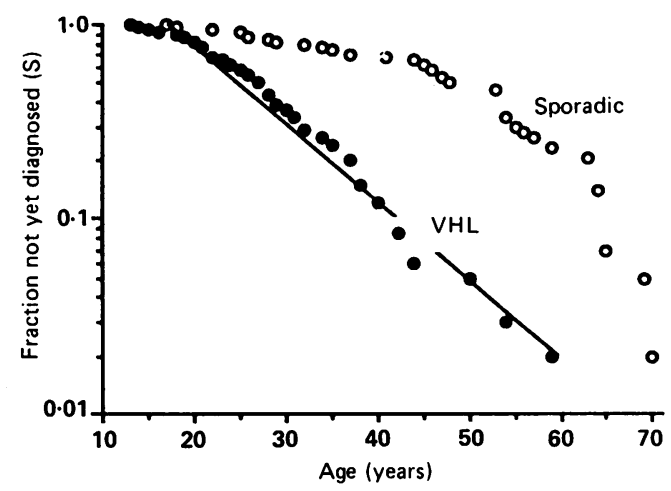

Figure 2 Age incidence of cerebellar haemangioblastoma in $V H L$ disease and in sporadic cases. For sporadic cases the data fitted a second order equation

$\left(\log _{10} S=0 \cdot 23-2 \cdot 78 \times 10^{-4} t^{2} r=0 \cdot 92\right)$ better than $a$ first order equation. For VHL disease a first order equation was fitted $\left(\log _{10} S=0.69-0.04 t r=0.99\right)$.

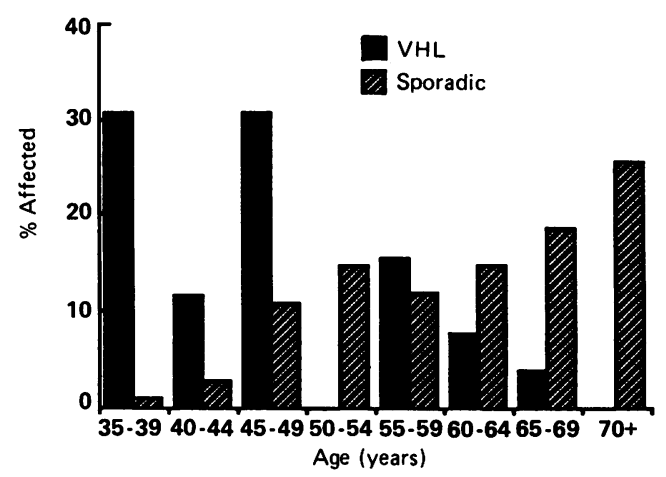

Figure 3 Age distribution of renal cell carcinoma in sporadic cases and in patients with VHL disease. 


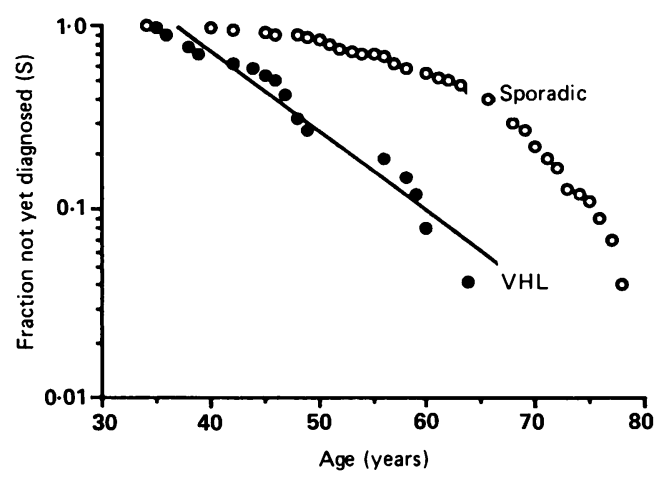

Figure 4 Age incidence of renal cell carcinoma in VHL disease and in sporadic cases. For sporadic cases the data fitted a second order equation $\left(\log _{10} S=0.67-2.93 \times 10^{-4} t^{2} r=0.94\right)$ better than a first order equation. For VHL disease a first order equation was fitted $\left(\log _{10} S=1.56-0.04 t r=0.97\right)$.

However, this difference in sex distribution between the two groups was not statistically significant $\left(\chi^{2}=2 \cdot 6, p>0 \cdot 1\right)$. Analysis of age incidence for VHL disease and sporadic renal cell carcinoma cases gave similar results to those seen for cerebellar haemangioblastoma. The relationship between $\log \mathrm{S}$ (proportion of cases not yet diagnosed) and $t$ (age) was best described by a first order equation for VHL disease tumours and a second order equation for sporadic tumours (fig 4). The linear regression equation for renal cell carcinoma in VHL disease $\left(\log _{10} S=1.56\right.$ $0.04 \mathrm{t}$ ) was similar to that obtained by Erlandssen et $a t^{6}$ for familial renal cell carcinoma $\left(\log _{10} S=1 \cdot 23-0 \cdot 036 \mathrm{t}\right)$.

\section{Discussion}

We found that both cerebellar haemangioblastoma and renal cell carcinoma occurring in VHL disease followed a single stage (somatic) mutation model originally described for bilateral retinoblastoma tumours by Knudson. ${ }^{1}$ Both sporadic cerebellar haemangioblastoma and renal cell carcinoma fitted a two stage mutation model. Erlandssen et at (using data from published studies) also fitted a two stage mutation model curve to the age incidence data for sporadic renal cell carcinoma. In Knudson's analyses of childhood retinoblastoma ${ }^{1}$ and Wilms' tumour ${ }^{3}$ the equations for the first and second order relationships $\left(\log \mathrm{S}=\mathrm{a}-\mathrm{kt}\right.$ and $\left.\log \mathrm{S}=\mathrm{a}-\mathrm{kt}^{2}\right)$ were constrained so that $a=0$. In contrast, we and others ${ }^{5}{ }^{6}$ who studied tumours which occur in adults found that $a>0$. The mathematical justification for this is clear, as a linear relationship between $\log S$ and $t$ could not be fitted if a was constrained to equal 0 . However, there is no primary biological reason why a should equal 0 for adult tumours. The value of a may be determined by the age dependent pattern of growth and differentiation for the particular cell type from which the tumour arises. For renal tumours there is no clinical overlap between the age at presentation of Wilms' tumour and renal cell carcinoma. For Wilms' tumour the opportunity for the second mutation only seems to occur in childhood. For renal cell carcinoma the opportunity for the second mutation may only occur in adulthood and it seems reasonable that the value of a should be different for the two tumours.

Between 30 and $40 \%$ of all cerebellar haemangioblastomas occur in patients with VHL disease, ${ }^{12} 13$ which is similar to Knudson's ${ }^{13}$ estimates that 35 to $40 \%$ of retinoblastoma cases and $38 \%$ of Wilms' tumours were inherited. The gene for VHL disease has been mapped to the short arm of chromosome 3 by genetic linkage analysis. ${ }^{14}$ Cerebellar haemangioblastomas in VHL disease patients show loss of heterozygosity for DNA markers on chromosome $3 \mathrm{p}{ }^{15}$ In these cases the loss of heterozygosity is on the chromosome with the wild type allele, which is compatible with the retinoblastoma model of tumourigenesis occurring in VHL disease. It is anticipated that sporadic cerebellar haemangioblastoma will also show loss of heterozygosity on $3 p$, but this has not yet been demonstrated.

The incidence of VHL disease is approximately 1 per 100000 . Renal cell carcinoma accounts for up to $3 \%$ of human cancers so that only a small proportion of all renal cancers are caused by VHL disease. Both sporadic renal cell carcinoma and tumours from VHL disease patients show loss of heterozygosity for chromosome $3 p$ markers, ${ }^{15-18}$ and cytogenetically visible deletions have also been reported. ${ }^{17} 1920$ Although these observations may seem compatible with the retinoblastoma model of tumourigenesis where sporadic renal cell carcinoma would result from somatic mutation of both alleles of the VHL disease gene, there is evidence that genes at loci other than the VHL gene are involved in the pathogenesis of sporadic renal cell carcinoma. Inherited renal cell carcinomas not caused by VHL disease have been associated with translocations involving the short arm of chromosome $3 .{ }^{21} 22$ A constitutional $t(3 ; 8)$ reciprocal translocation was associated with an $80 \%$ risk of renal cell carcinoma up to the age of 60 in one family. ${ }^{21}$ The translocation breakpoint was at 3p14.2. ${ }^{23}$ The most likely location for the VHL locus is telomeric to this at $3 \mathrm{p} 25-\mathrm{p} 26 .{ }^{24}$ There are other examples of familial renal cell carcinoma not associated with VHL disease or chromosomal abnormalities. ${ }^{25}$ Current data would seem to suggest that at least two loci on the short arm of chromosome 3 may be involved in the pathogenesis of renal cell carcinoma, one locus being proximal, and another the locus for VHL disease, which appears to map to the distal part of $3 p$.

Our data present statistical evidence for VHL disease being caused by an inherited mutation of a 
'tumour suppressor gene' of the retinoblastoma type. Sporadic cerebellar haemangioblastoma and some sporadic renal cell carcinoma may result from somatic mutations involving both alleles of the VHL disease gene. Further molecular genetic studies will help to confirm or refute this hypothesis.

We thank the National Kidney Research Fund for financial support, and the many colleagues who provided details of their patients.

1 Knudson AG Jr. Mutation and cancer: statistical study of retinoblastoma. Proc Natl Acad Sci USA 1971;68:820-3.

2 Cavanee WK, Dryja TP, Phillips RA, et al. Expression of recessive alleles by chromosomal mechanisms in retinoblastoma. Science 1983;305:779-884.

3 Knudson AG Jr, Strong LC. Mutation and cancer: a model for Wilms' tumor of the kidney. $\mathcal{F}$ Natl Cancer Inst 1972;48: 313-24.

4 Knudson AG Jr, Strong LC. Mutation and cancer: neuroblastoma and phaeochromocytoma. Am $\mathcal{F}$ Hum Genet 1972;24: 514-32.

5 Jackson CE, Block MA, Greenawald KA, Tashjian AH. The twomutational event theory in medullary thyroid cancer. Am $\mathcal{F}$ Hum Genet 1979;31:704-10.

6 Erlandssen R, Boldog F, Sumegi J, Klein G. Do human renal cell carcinomas arise by a double-loss mechanism? Cancer Genet Cytogenet 1988;36:197-202.

7 Melmon KL, Rosen SW. Lindau's disease. Am 7 Med 1964;36: 595-617.

8 Horton WA, Wong V, Eldridge R. Von Hippel-Lindau disease. Arch Intern Med 1976;136:769-77.

9 Lamiell JM, Sakazar FG, Hsia YE. Von Hippel-Lindau disease affecting 43 members of a single kindred. Medicine (Baltimore) 1989;68:1-29.

10 Levine E, Weigel JW, Collins DL. Diagnosis and management of asymptomatic renal cell carcinomas in von Hippel-Lindau syndrome. Urology 1983;21:146-50.

11 Maher ER, Harris R, Yates JRW, Ferguson-Smith MA. Clinical features and natural history of von Hippel-Lindau disease. $\mathcal{F}$ Med Genet 1989;26:600-1A.

12 Jeffreys $\mathbf{R}$. Clinical and surgical aspects of posterior fossa haemangioblastomata. F Neurol Neurosurg Psychiatry 1975;38: 105-11.

13 Huson SM, Harper PS, Hourihan MD, Cole G, Weeks RD, Compston DAS. Cerebellar haemangioblastoma and von Hippel-Lindau disease. Brain 1986;109:1297-310.

14 Seizinger BR, Rouleau GA, Ozelius LJ, et al. Von HippelLindau disease maps to the region of chromosome 3 associated with renal cell carcinoma. Nature 1988;332:268-9.

15 Tory $\mathrm{K}$, Brauch $\mathrm{H}$, Lineham $\mathbf{M}$, et al. Specific genetic change in tumors associated with von Hippel-Lindau disease. 7 Nat Cancer Inst 1989;81:1097-101.

$16 \mathrm{Zbar} \mathrm{B}$, Brauch $\mathrm{H}$, Talmadge $\dot{\mathrm{C}}$, Linehan $\mathrm{M}$. Loss of alleles of loci on the short arm of chromosome 3 in renal cell carcinoma. Nature 1987;327:721-4.

17 Kovacs G, Erlandssen R, Boldog F, et al. Consistent chromosome $3 p$ deletion and loss of heterozygosity in renal cell carcinoma. Proc Natl Acad Sci USA 1988;85:1571-5.

18 Van der Hout AH, Kok K, van den Berg A, Oosterhuis JW, Carritt B, Buys CHCM. Direct molecular analysis of a deletion of $3 p$ in tumours from patients with sporadic renal cell carcinoma. Cancer Genet Cytogenet 1988;32:281-5.

19 Yoshida MA, Ohyashiki K, Ochi H, et al. Cytogenetic studies of tumor tissue from patients with nonfamilial renal cell carcinoma. Cancer Res 1986;46:2139-47.

20 King CR, Schimke RN, Arthur T, Davoren B, Collins D. Proximal $3 p$ deletion in renal cell carcinoma cells from a patien with von Hippel-Lindau disease. Cancer Genet Cytogenet 1987; 27:345-8.

21 Cohen AJ, Li FP, Berg S, et al. Hereditary renal-cell carcinoma associated with a chromosomal translocation. $N$ Engl $7 \mathrm{Med}$ 1979;301:592-5.

22 Pathak S, Strong LC, Ferrell RE, Trindade A. Familial renal cell carcinoma with a 3;11 chromosome translocation limited to tumor cells. Science 1982;217:939-41.

23 Wang N, Perkins KL. Involvement of band $3 p 14$ in $t(3 ; 8)$ hereditary renal carcinoma. Cancer Genet Cytogenet 1984;11: 479-81.

24 Seizinger BR, Farmer G, Haines J, et al. Isolating the gene(s) for von Hippel-Lindau disease and renal cell carcinoma. Am $\mathcal{f}$ Hum Genet 1989;45(suppl):32A.

$25 \mathrm{Li} \mathrm{FP,} \mathrm{Marchetto} \mathrm{DJ,} \mathrm{Brown} \mathrm{RS.} \mathrm{Familial} \mathrm{renal} \mathrm{cell} \mathrm{carcinoma.}$ Cancer Genet Cytogenet 1982;7:271-5. 